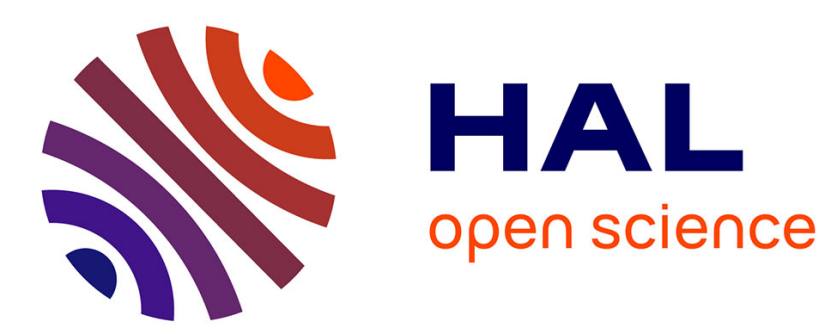

\title{
TRANSDUCTEURS ÉLECTROACOUSTIQUES ET ÉLECTROMÉCANIQUES ET LEURS CIRCUITS ÉQUIVALENTS
}

Z. Skvor

\section{- To cite this version:}

Z. Skvor. TRANSDUCTEURS ÉLECTROACOUSTIQUES ET ÉLECTROMÉCANIQUES ET LEURS CIRCUITS ÉQUIVALENTS. Journal de Physique IV Proceedings, 1992, 02 (C1), pp.C1349-C1-356. 10.1051/jp4:1992175 . jpa-00251247

\section{HAL Id: jpa-00251247 https://hal.science/jpa-00251247}

Submitted on 1 Jan 1992

HAL is a multi-disciplinary open access archive for the deposit and dissemination of scientific research documents, whether they are published or not. The documents may come from teaching and research institutions in France or abroad, or from public or private research centers.
L'archive ouverte pluridisciplinaire HAL, est destinée au dépôt et à la diffusion de documents scientifiques de niveau recherche, publiés ou non, émanant des établissements d'enseignement et de recherche français ou étrangers, des laboratoires publics ou privés. 


\title{
TRANSDUCTEURS ELECTROACOUSTIQUES ET ELECTROMECANIQUES ET LEURS CIRCUITS ÉQUIVALENTS
}

\author{
Z. ŚKVOR \\ Faculté Electrotechnique, Université Technique Tchèque de Prague, Technická, 16627 Praha 6 \\ Czechoslovakia
}

\begin{abstract}
Electromechanical and electroacoustic transducers and their equivalent circuits.

This paper describes analog models of particular types of electroacoustic transducers including electrostatic transducers in spiral form. The description is based on the electromechanical analogies with lumped elements systems and focuses on diaphragms, bands and air-grap. Some experimental results will be presented.
\end{abstract}

\section{Introduction}

\begin{abstract}
Parmi les nombreux transducteurs électromécaniques et électroacoustiques réciproques, certains possèdent une propriété particulière : un même élément joue à la fois le rôle électrique actif et le rôle principal dans la transquction mécano-acoustique. Dans la famille des transducteurs basés sur un champ électrique, ce sont les transducteurs électrostatiques et piézoplastiques pour lesquels la membrane (ou la bande) joue ce double rôle. Dans le cas des transducteurs basés sur le champ magnétique, ce sont les transducteurs électrodynamiques à ruban.
\end{abstract}

La description de ce type de transducteur au moyen de schémas équivalents analogiques permet le plus souvent de décrire avec une précision suffisante leurs propriétés électroacoustiques. Cette description basée sur des analogies électromécaniques et électroacoustiques des éléments répartis offre un instrument de synthèse et d'optimisation.

Plusieurs auteurs ont cherché la description du système membraneentrefer [1]-[8] et piston-entrefer dans diverses conditions géométriques et thermodynamiques. Les travaux les plus récents décrivent un système possédant une géométrie simple et composé d'une membrane mince - d'un entrefer - d'une électrode fixe, en considérant le milieu gazeux comme un fluide visco-thermique réel. 


\section{Modélisation de 1 'entrefer}

Très souvent la géométrie de l'électrode fixe (dans le cas du microphone électrostatique par exemple) est relativement complexe en raison de la présence de perforations réparties de façon non uniforme, et il faut trouver des méthodes particulières qui autorisent une description suffisamment précise de son comportement [5], [9], [10], [11].

Dans les références [10] et [12], on donne la description d'une méthode de visualisation de l'écoulement gazeux entre deux plaques, l'une d'entre elles modélisant une électrode fixe perforée et l'autre une membrane fonctionnant en mode piston. Un écoulement gazeux entre deux électrodes peut être décrit par un simple modèle hydrodynamique. Par analogie une suspension aqueuse de colorant en poudre mise entre deux plaques (de simple plexiglass par exemple) forme une couche mince simulant un milieu gazeux incompressible. En écartant les plaques suivant une direction normale, les particules de colorant se rassemblent formant des figures qui représentent les frontières entre les domaines d'écoulement contigüs entourant chaque perforation de l'électrode fixe.

Sur la Fig. 1 deux exemples de figures obtenues sur deux plaques qui. possèdent des répartitions différentes de perforations sont présentés.

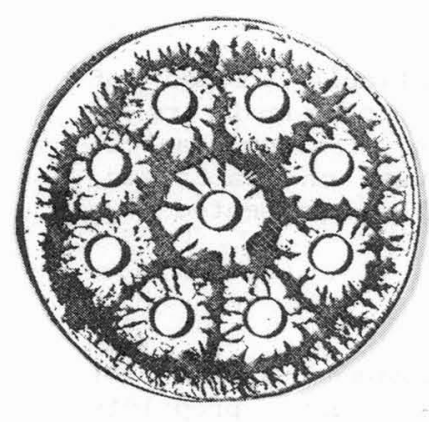

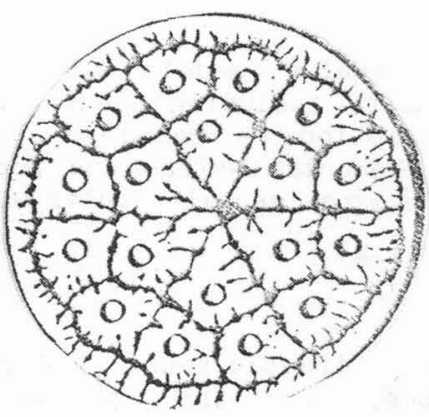

Fig. 1

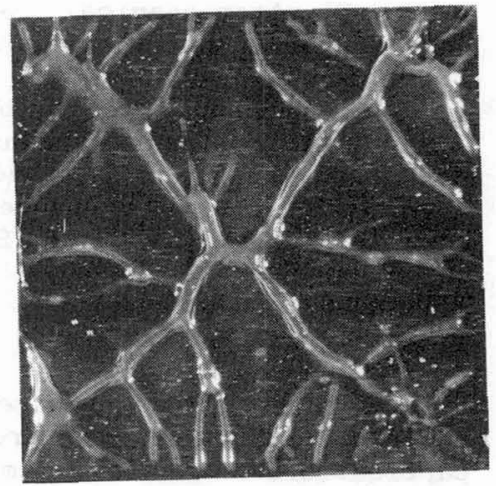

Fig. 2

Fig. 1 : Modèle hydrodynamique de l'entrefer de transducteur électrostatique (Hydrodynamic model of an air-gap of an elecrostatic transducer with general perforation patern in the fixed electrode)

Fig. 2 : Modèle hydrodynamique de l'entrefer - Membrane carrée (Hydrodynamic model of an air-gap between a rectangular diaphragm and a fixed electrode)

Cette description facilite la recherche de modèle, en retenant 1 'hypothèse de l'incompressibilité pour le milieu visqueux, permettant le calcul des pertes visqueuses et par suite l'obstention de résistance acoustique de charge de la membrane vibrante en mode piston [9], [10], $[11],[12]$.

Sur la Fig. 2, nous présentons un modèle de l'entrefer de la membrane carrée (vibrant en mode piston). Les figures présentent une "forme diagonale" qui divise l'entrefer en quatre domaines triangulaires identiques. 
Les modèles de l'entrefer, présentés en [11] et [12], facilitent la description des systèmes de transducteur à membranes et bandes. Un entrefer parallélépipédique, d'épaisseur $h$, de largeur $b$, et de longueur $\ell$ (Fig.3) peut être décrit de manière simple par un quadripôle (Fig.4).
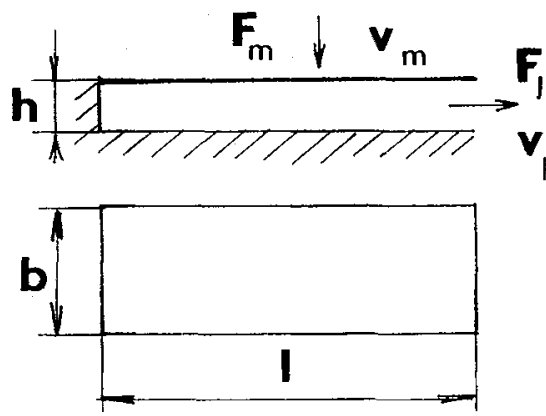

Fig. 3

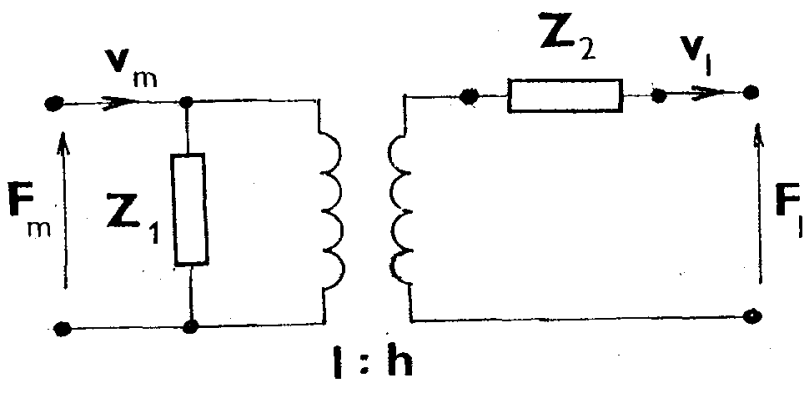

Fig. 4

Fig. 3 : Entrefer parallélépipédique

(Air-gap under rectangular diaphragm vibrating in piston-like mode)

Fig. 4 : Schéma analogique de l'entrefer parallélépipédique

(Analog network of an air-gap under a rectangular diaphragm)

Sur la Fig. 4, les impédances $Z_{1}$ et $Z_{2}$ sont égales à :

$$
z_{1}=\frac{1}{j \omega} \frac{k}{b \ell} \quad \text { (1) } \quad z_{2}=4 \mu \frac{b \ell}{4 h}+j \omega \frac{2}{3} b \ell h
$$

De l'équation de Navier-Stokes, pour un fluide visqueux non compressible, on peut déduire l'impédance de I'entrefer sous la membrane carrée aux basses fréquences dans chacun des quatre domaines triangulaires. Sur la Fig.5, on présente une membrane carrée de côté a et séparée de I'électrode fixe d'une distance h. L'impédance de l'entrefer rapportée sur la membrane $\mathrm{F}_{\mathrm{m}} / \mathrm{v}_{\mathrm{m}}=\mathrm{z}_{2}$ est égale à :

$$
Z_{2}^{\prime}=\frac{3}{8} \mu \frac{a^{4}}{h^{3}}+j \omega \frac{6}{5.32} \frac{a^{4}}{h}
$$

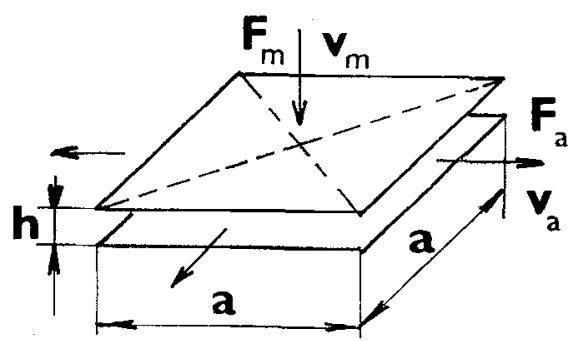

$$
z_{2}=\left(\frac{4 h}{a}\right)^{2} z_{2}
$$

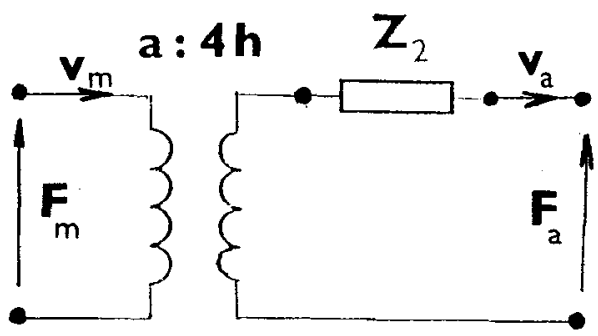

Fig. 5 : Entrefer sous une membrane carrée et son schéma analogique (Air-gap under square diaphragm vibrating in piston-like mode and its analog network) 


\section{Microphone à membrane carrée}

A partir des résultats précédents, on peut formuler un schéma équivalent pour les microphones à membrane carrée, présenté sur la Fig. 6 , où

$$
\begin{array}{ll}
C_{0}=\frac{\varepsilon_{0} a^{2}}{h} & \text { est la capacitance de transducteur } \\
k_{b} & \text { le rapport de transduction } \\
U_{0} & \text { la tension de polarisation }
\end{array}
$$

$R_{m}^{\square}=\operatorname{Re} Z_{2}$ (éq. (3)) la résistance mécanique de l'entrefer

$M_{m}^{\square}=\operatorname{Im} Z_{2}$ (éq. (3)) la masse de l'entrefer

$c_{a x}=\frac{\gamma p_{0}}{v_{x}} \quad$ la souplesse acoustique de la cavité extérieure (volume $v_{x}$ ) $c=\frac{4}{\pi^{4} v} \quad \begin{aligned} & \text { la souplesse de la membrane }[12], \text { ( } v \text { est la tension mécanique } \\ & \text { de la membrane) }\end{aligned}$

$m=\frac{\pi^{2} m_{1} a^{2}}{4}$

la masse de la membrane $[12]$ ( $m_{1}$ est la masse surfacique de la membrane)

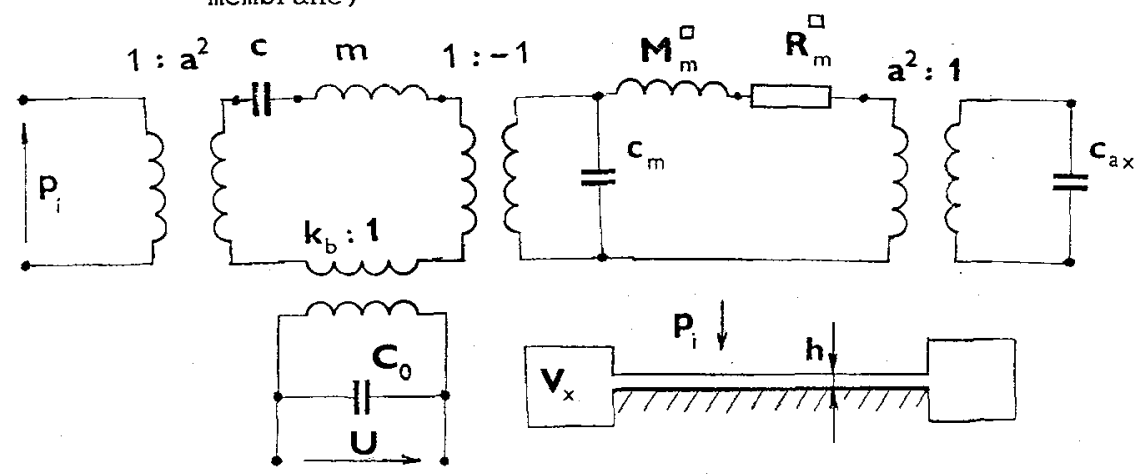

Fig. 6 : Schéma équivalent analogique du microphone à membrane carrée (Equivalent network of a microphone with a square diaphragm)

La masse apparente de la membrane $m$ et sa souplesse sont celles qui correspondent au mode fondamental de la membrane carrée [12]. Le schéma de la fig. 6 donne des résultats pour la courbe de sensibilité qui sont en bon accord avec ceux obtenus par la mesure sur un microphone miniature présenté en $[8]$.

\section{Transducteur électrostatique en spirale}

Un transducteur électrostatique en spirale [13] présenté sur la fig. 7 est réalisé par une paire d'électrodes fixes $(5,7)$, par une paire de membranes $(6,8)$ enroulées sur un noyau cylindrique (9). La distance entre les électrodes est déterminée par quatre bandes séparatrices $(1,2,3,4)$ en matériau isolant. Le transducteur a une forme finale cylindrique, les faces rayonnantes coïncidant avec les bases du cylindre. La surface active de la membrane est de un à deux ordres de grandeur plus importante que la surface des fentes de sortie. 


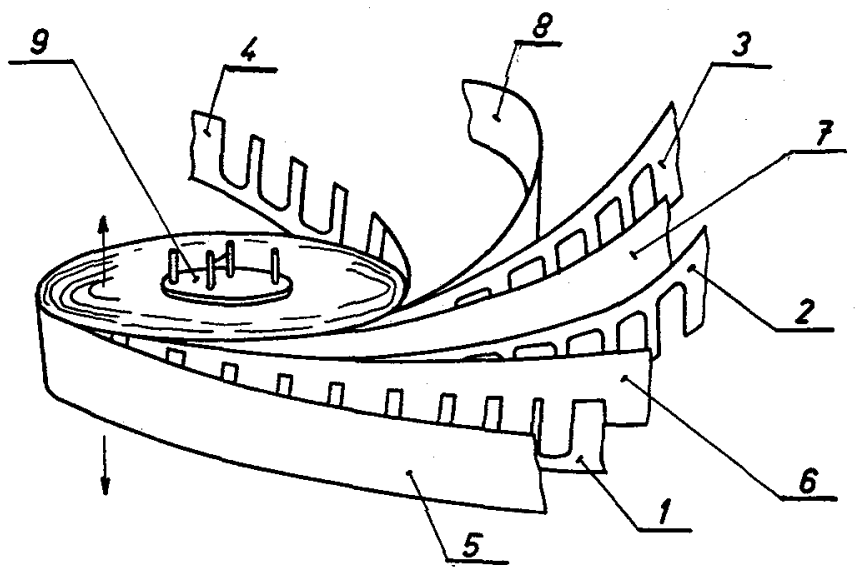

Fig. 7

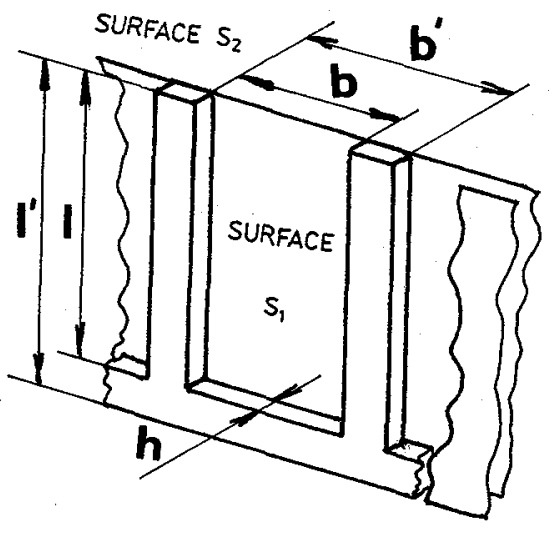

Fig. 8

Fig. $7_{y}$ : Transducteur électrostatique en spirale

(Electrostatic spiral transducer)

Fig. 8 : Elément de transducteur en spirale

(An element of a spiral transducer)

L'illustration de la Fig. 8 représente un élément de la bande de longueur $\ell$ ' et de largeur ' $\mathbf{b}^{\prime}$ dont la partie mobile est de longueur $\ell$ et de largeur $\mathbf{b}$. L'entrefer a une forme parallélépipédique qui peut être modélisé par le schéma de la Fig.4, à partir duquel on peut obtenir le schéma équivalent complet d'un transducteur en spirale fonctionnant en émetteur.

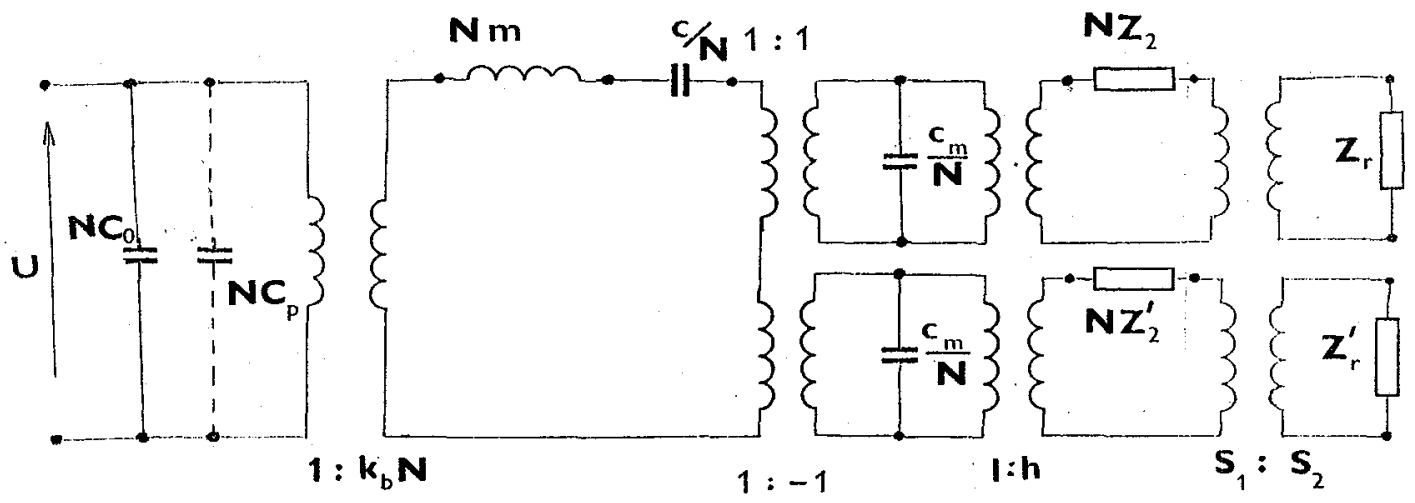

Fig. 9 : Schéma équivalent du transducteur électrostatique en spirale (Equivalent network of a electrostatic spiral transducer) 
Sur la Fig.9, les définitions et expressions des éléments sont les suivantes :

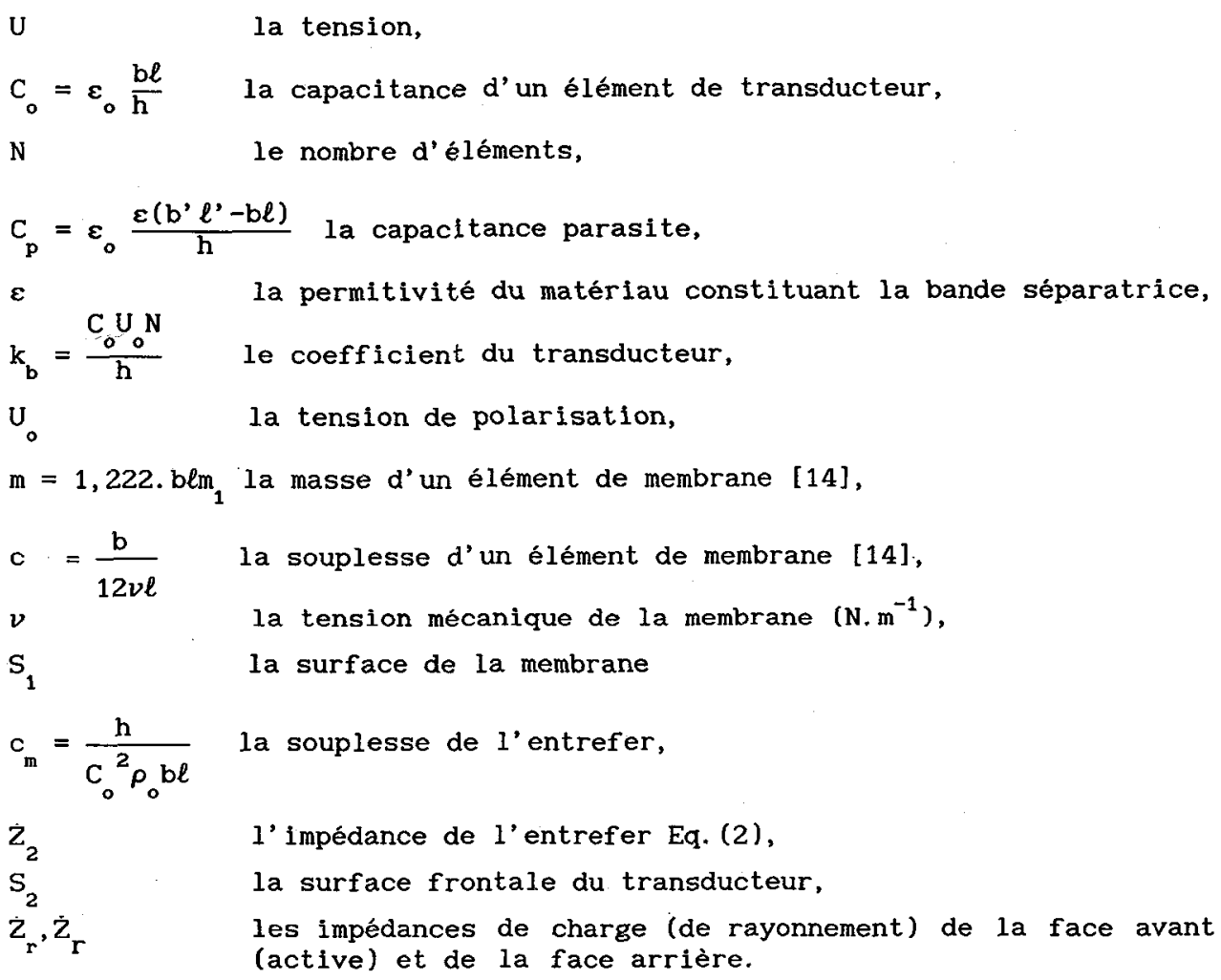

\section{Exemples d'application de transducteur en spirale}

Les transducteurs de ce type peuvent servir comme tweeter, avec un pavilion ou en rayonnement direct. Sur les Fig. 10 et 11 sont présentés respectivement les dimensions intérieures du transducteur et un transducteur monté dans un pavillon exponentiel.

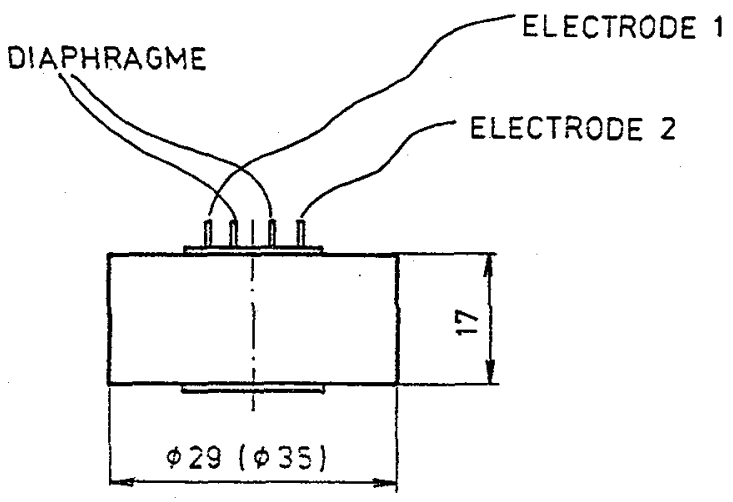

Fig. 10 : Transducteur en spirale (Spiral transducer) 


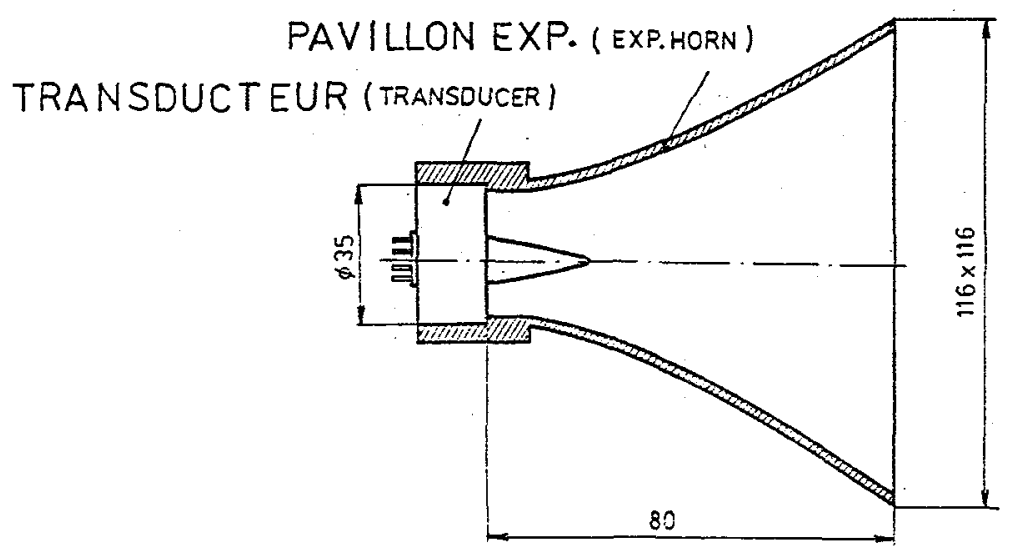

Fig. 11 : Transducteur en spirale comme un tweeter avec un pavilion exponentiel

(Spiral transducer as a tweeter with an exponential horn)

Sur la Fig. 12, on donne les courbes de réponse du transducteur en spiral, de diamètre externe $\varnothing 35 \mathrm{~mm}$, utilisé respectivement comme tweeter sans pavillon et avec un pavillon exponentiel (de fréquence de coupure $800 \mathrm{~Hz}$ ). Dans le cas du transducteur muni d'un pavillon, on remplace dans la Fig. 9 l'impédance de rayonnement $Z_{r}$ par un quadripôle en $\Pi$ en cascade avec un transformateur analogue [12].

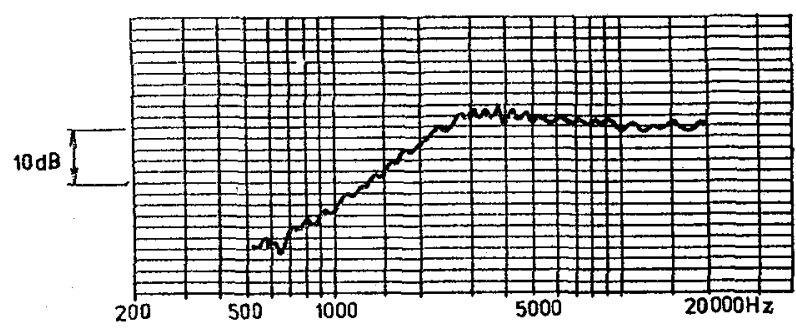

$U_{0}=380 \mathrm{~V}, u=2 \times 150 \mathrm{~V}$. distance $l=0,5 \mathrm{~m}$

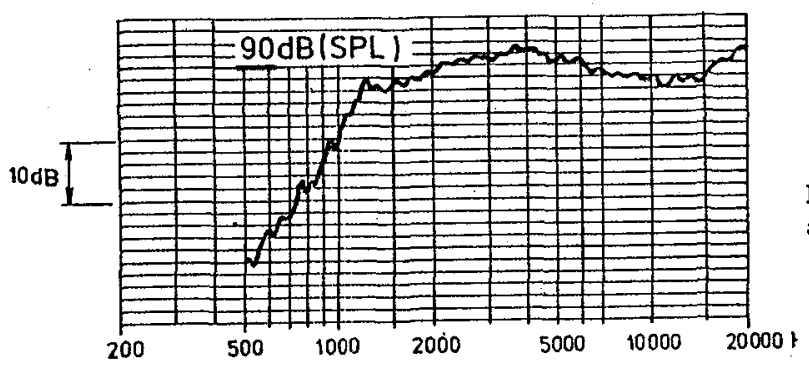

Haut-parleur électrostatique sans pavillon (Electrostatic tweeter without horn)
Haut-parleur électrostatique avec un pavillon exponentiel (Electrostatic tweeter with horn)

Fig. 12 : Réponses en fréquence du transducteur électrostatique en spirale (Frequency response of electrostatic spiral transducer as a tweeter) 


\section{Conclusion}

Nous avons présenté des modèles simplifiés de l'entrefer formant diverses parties de transducteurs électroacoustiques, dans lesquels les membranes où les bandes sont actives en terme de transduction électromécanique et même mécano-acoustique.

Par leur capacité à tenir compte de tous les phénomènes essentiels, dont les formes géométriques complexes, la dirsipation par viscosité de cisaillement, et du fait de leur simplicité fillale puisque traduisible en forme de circuits électriques équivalents, ces modèles facilitent une étude synthétique efficace, et corrélativement sont d'une grande utilité pour la réalisation de nouveaux types de transducteurs, parmi lesquels ceux exposés dans ce papier. Les solutions apportées à la réalisation des transducteurs particuliers présentés au-dessus sont totalement transposables au cas des transducteurs piézo-plastiques [15] et électrodynamiques à ruban. Les propriétés obtenues rendent ces types de transducteurs éligibles pour des applications particulières.

\section{Bibliographie}

[1] Robey D. H., J. Acoust. Soc. Am. 26 (1954), 740-745.

[2] Merhaut J., Acustica 19, 5, (1967/68), 284-292.

[3] Warren J.E., Brzezinski A., Hamillon J., J. Acoust. Soc. Am. 52, (1972), 711-719.

[4] Warren J.E., Brzezinski A., Hamillon J., J. Acoust. Soc. Am. 54, (1973), 1201-1213.

[5] Zuckerwar A. J., J. Acoust. Soc. Am. 64, 5, (1978), 1278-1285.

[6] M. Bruneau et al, Wave Motion II, (1989), 441-451.

[7] Plantier G., Bruneau M., J. Acoustique 3 (1990), 243-250.

[8] Bruneau M., Bruneau A.M., Hamery P., Molélisation des microphones miniatures, 2ème Congrès Français d'Acoustique, 1992, Arcachon.

[9] Škvor Z., Acustica 19, 1967/68, 5, 295-299.

[10] Škvor Z., Hochfrequenz und Elektroakustik 80, 3, Leipzig, 1971, 85-92.

[11] Merhaut J., Škvor Z., Proc. IREE, 37, 3, (3/1976), 63-68. .

[12] Škvor Z., Vibrating sytsems and their equivalent circuits, Elsevier Publ. Sc. Comp. 1992, Amsterdam.

[13] Škvor Z., Proceeding of the $8^{\text {th }}$ Congress ICA, London, July 1974, p. 680 .

[14] Merhaut J., Theory of electroacoustics, Mc Graw Hill Int. Book. Comp., New-York, 1981.

[15] Škvor Z., Kadlec Fr., Bruneau A.M., Lotton P., Contribution to the theory of piezoélectrical transducer, Workshop 92, Czech Technical University in Prague, 20-24, January, 1992 (to be published). 